\title{
Hydrocera triflora, die weitgehend unbekannte Schwesterart der Springkräuter (Impatiens)
}

Stefan Abrahamczyk, Bernd Reinken \& Klaus Michael Neumann

\section{Abstract}

Hydrocera is a monotypic genus, which is sister to the touch-me-not balsams (Impatiens). We describe its dispersal and give hints for its cultivation and propagation.

\section{Zusammenfassung}

Hydrocera ist eine monotypische Gattung und Schwester zu den Springkräutern (Impatiens). Wir beschreiben ihren Ausbreitungsmechanismus und geben Hinweise für ihre Kultivierung und Vermehrung.

\section{Verbreitung}

Zur Familie der Springkrautgewächse (Balsaminaceae) gehören zwei Gattungen: die Springkräuter (Impatiens) mit weit mehr als $1000 \mathrm{Ar}$ ten und die monotypische Gattung Hydrocera (Janssens et al. 2012) mit der einzigen Art $H y$ drocera triflora. Während die Springkräuter hauptsächlich in den Bergwäldern der Tropen und Subtropen der Alten Welt vorkommen, besiedelt Hydrocera triflora die tropischen Tieflandsümpfe Süd- und Südostasiens. Ihr Verbreitungsgebiet reicht von Sri Lanka und Indien im Westen bis nach Sulawesi im Osten und Südchina im Norden und schließt auch das indomalayische Festland mit ein (GREY-WiLson 1980, Fischer 2004).

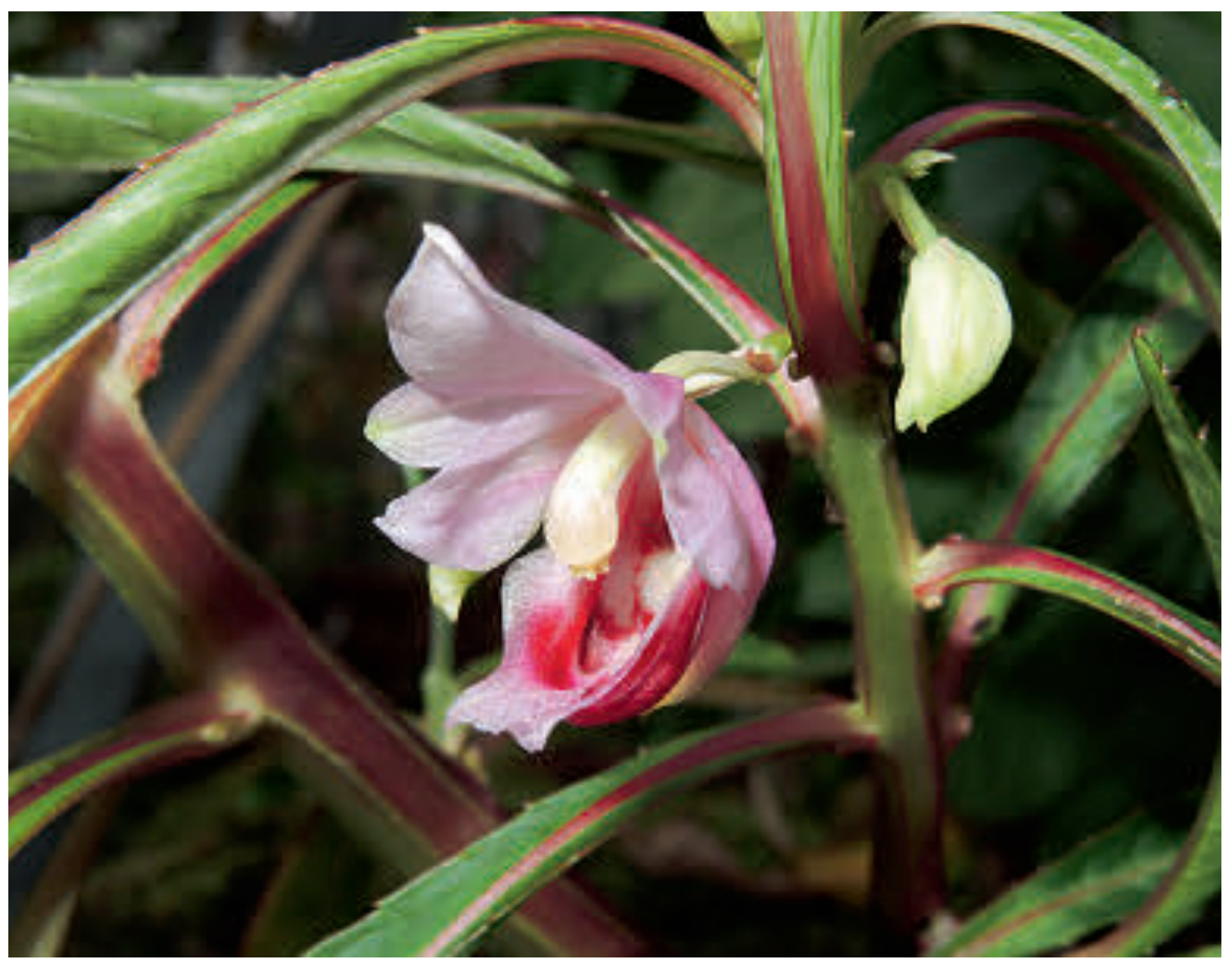




\section{2. Äußeres Erscheinungsbild}

Hydrocera ist eine krautige, sukkulente, ausdauernde, halbaquatische Sumpfpflanze. Sie bildet gespornte, fünfzählige, rosa Blüten mit kaminroten Streifen. Ihre Kron- und Kelchblätter sind frei, wodurch sich Hydrocera von den meisten Springkrautarten unterscheidet (GreyWilson 1980). Das einzige echte Gattungsmerkmal ist aber ihre Frucht (Janssens et al. 2012). Im Gegensatz zu den explosiven Kapselfrüchten der Springkräuter besitzt Hydrocera dunkelrote, fleischige Scheinbeeren mit einem harten Endocarp. Sobald sie die Fruchtreife erreichen, fallen diese Früchte schon bei der geringsten Berührung $\mathrm{ab}$, was gegen die scheinbar offensichtliche Verbreitung durch Vögel spricht (eigene Beobachtung). Im Wasser schwimmen die Früchte einige Zeit und öffnen sich nach 5 bis 15 Tagen fünfklappig. Nach dem Öffnen sinkt die Frucht nach kurzer Zeit ab. Die fünf Samen, die in eine schleimige Schicht gehüllt sind, schwimmen, von zwei Luftsäcken getragen, oft noch kurzzeitig weiter (FISCHER 2004). Aufgrund dieser Anpassungen ist bei Hydrocera von Wasserverbreitung auszugehen (FISCHER 2004).

\section{Kultur von Hydrocera}

Die Kultur von Hydrocera ist relativ leicht. Die Pflanze sollte ständig direkten Wasserkontakt über die Wurzeln haben. In den Botanischen Gärten von Bonn ist Hydrocera triflora im Victoriahaus in einem Sumpfbankbeet unter einer Pachira aquatica im Halbschatten ausgepflanzt und steht einige Zentimeter oberhalb der Wasserfläche, wo sie prächtig gedeiht. Weitere Pflanzen, die in Töpfen im Anzuchtbereich kultiviert werden, stehen entweder unbeschattet in wassergefüllten Untersetzern oder auf umgedrehten leeren Töpfen im Wasserbecken, sodass sich Teile des Wurzelbereichs unterhalb der Wasserlinie befinden. Die optimale Temperatur für die Kultivierung von Hydrocera be-

Abb. 1 (Seite 16): Blüte von Hydrocera triflora.

Abb. 2 (oben): Frucht von Hydrocera triflora.

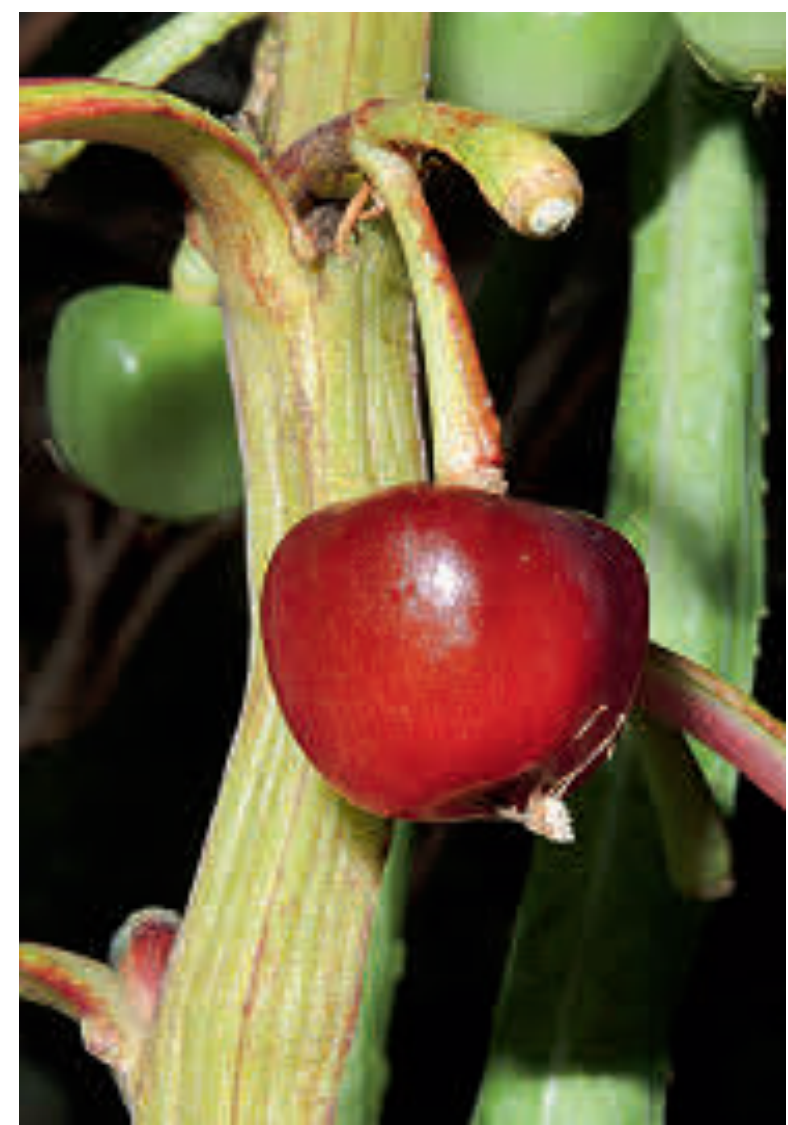

trägt um $25^{\circ} \mathrm{C}$ Celsius. Die Luftfeuchtigkeit liegt bei $60-80 \%$, toleriert werden auch geringfügig tiefere Temperaturen und Luftfeuchtigkeit. Während einer längeren Beprobungsphase im Forschungsgewächshaus bei Tagestemperaturen von $17^{\circ} \mathrm{C}$ und einer Nachtabsenkung auf $15^{\circ} \mathrm{C}$ verschlechterte sich der Zustand der Pflanzen allerdings deutlich, sodass ein Absterben der Pflanzen drohte. Während der Wintermonate ist es empfehlenswert, Hydrocera zusätzlich zu beleuchten.

Das Substrat, das wir für die Kultur von Hydrocera verwenden, besteht aus 70-80\% Einheitserde ED73 und ca. 20-30\% Sand. Einheitserde ED 73 besteht zu $70 \%$ aus Torf und $\mathrm{zu} 30 \%$ aus Ton mit einem pH-Wert von ca. 5,8 und ist aufgedüngt mit einem N-P-KDünger 14:16:18; außerdem enthält es einen langsam wirkenden Dünger mit einer N-P-KKonzentration von 20:10:15. Hydrocera ist unempfindlich gegenüber Krankheiten und Schädlingen, wobei betont werden muss, dass die sehr wüchsigen Pflanzen regelmäßig umgetopft werden sollten. 


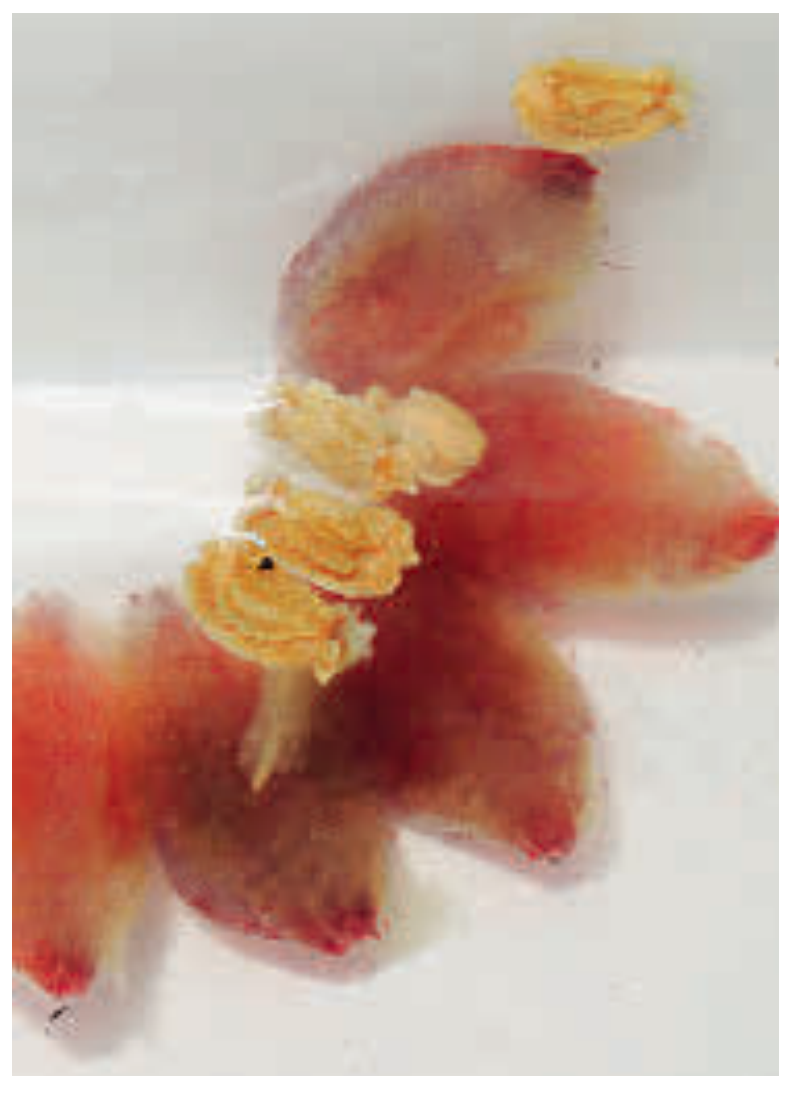

\section{Vermehrung von Hydrocera}

Die Vermehrung von Hydrocera kann sowohl vegetativ als auch generativ erfolgen. Die leichteste Methode, Hydrocera zu vermehren, besteht darin, Stecklinge bei einer Temperatur von ca. $25^{\circ} \mathrm{C}$ in ein Wasserglas zu stellen. Innerhalb weniger Wochen sind diese bewurzelt und können getopft werden.

Eine andere Möglichkeit ist es, die Scheinbeeren im reifen, dunkelroten Zustand zu ernten und für einige Tage bzw. Wochen in ein Wasserbad zu legen. Die Frucht weicht auf und entlässt die Samen nach 5-15 Tagen. Sobald die „reifen“ Samen auf den Grund abgesunken sind, können sie ausgesät werden. Um diesen Prozess zu beschleunigen, kann man das Fruchtfleisch der Scheinbeere vorsichtig zerdrücken und warten, bis die Samen absinken. Zur Aussaat kann das gleiche Substrat wie oben beschrieben verwendet werden. In unregelmäßigen Abständen von einer bis drei Wochen keimen die ersten Pflänzchen, wobei die Zeit bis zur Keimung kürzer wird, je länger die Samen im Wasser gelegen haben. Nach der Keimung muss sofort pikiert werden.

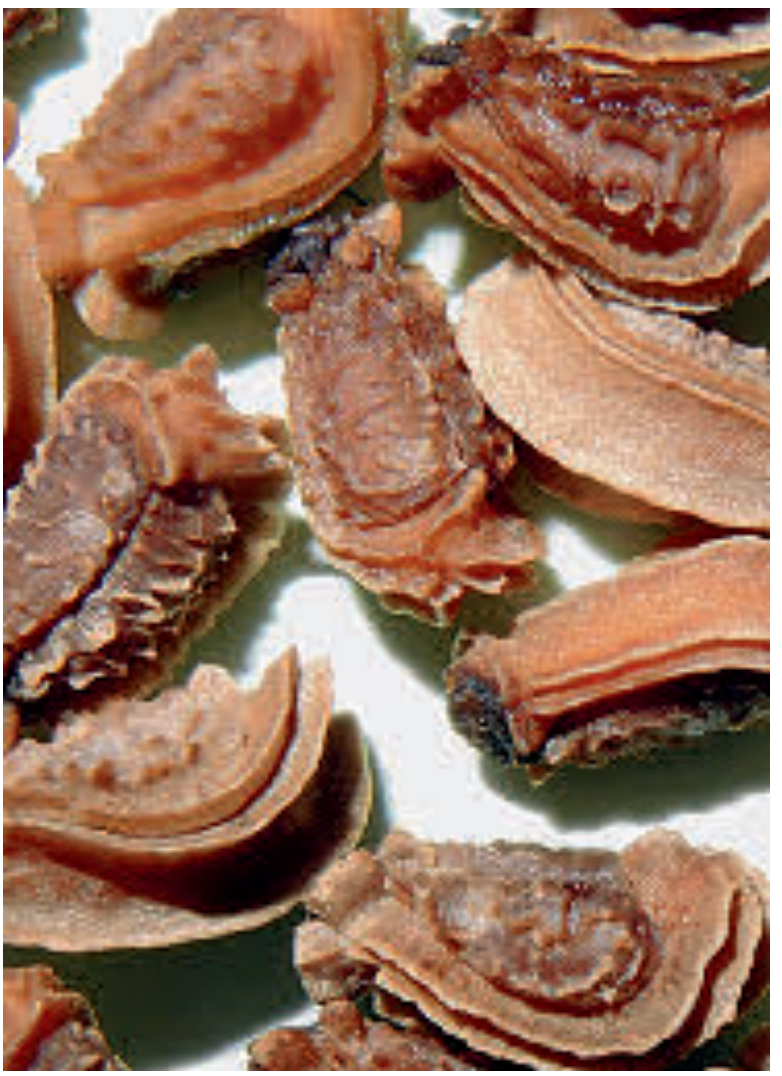

\section{Dank}

Wir danken Wolfram Lobin, Bonn, für das Korrekturlesen des Manuskriptes und Steven Janssens, Meise, für das Bereitstellen der Pflanzen.

\section{Literatur}

Fischer, E. 2004: Flowering plants. Dicotyledons. Balsaminaceae (pp. 20-25).- Berlin, Heidelberg. Grey-Wilson, C. 1980: Hydrocera triflora.

Its floral morphology and relationship with Impatiens: Studies in Balsaminaceae V. - Kew Bull. 35: 213-219. Janssens, S. B., Smets, E. F. \& VRijdaghs, A. 2012: Floral development of Hydrocera and Impatiens reveals evolutionary trends in the most early diverged lineages of the Balsaminaceae. - Ann. Bot. 109: 1285-1296.

Abb. 3 (links): Geöffnete Frucht.

Abb. 4 (rechts): Samen von Hydrocera triflora. 